\title{
Psicología
}

Artículo original

\section{Depresión mayor en población general de Envigado (Colombia): prevalencia y factores asociados}

\section{Major depression in the general population of Envigado (Colombia): prevalence and associated factors}

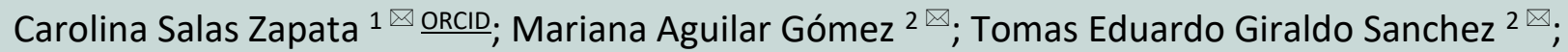
María Camila Muñoz Rua ${ }^{2 \otimes}$; Alejandra Torres Blandón ${ }^{2 \otimes}$; Alejandro Uribe Castaño ${ }^{2}$; Alejandra Uribe Quintero ${ }^{\boxplus}$

${ }^{1}$ Magister en Epidemiología. Docente investigadora, Grupo de investigación en Salud Mental, Universidad CES, Colombia.

2 Estudiante de Medicina, Universidad CES, Colombia.

Fecha correspondencia:

Recibido: noviembre 1 de 2019

Aceptado: junio 15 de 2021

Forma de citar:

Salas, C., Aguilar, M., Giraldo, T., Muñoz, M., Torres, A., Uribe, A., \& Uribe, A. (2021). Depresión mayor en población general de Envigado (Colombia): prevalencia y factores asociados. Rev. CES Psico, 14(3), 117-133. https://dx.doi.org/10.21615/ cesp. 5283

\section{Open access}

(C) Derecho de autor

Licencia creative commons

Ética de publicaciones

Revisión por pares

Gestión por Open Journal System

DOI: $10.21615 /$ cesp.5283

ISSNe: 2011-3080

Publica con nosotros

\section{Resumen}

Introducción: La depresión afecta a más de 300 millones de personas en el mundo, es la principal causa de discapacidad y contribuye de forma importante a la carga mundial de morbilidad. Objetivo: Determinar la prevalencia y algunos factores personales, sociales y familiares asociados al trastorno de depresión mayor en población de 15 a 65 años del municipio de Envigado (Colombia) en el año 2017. Método: Estudio transversal con intención analítica, a partir de la información de 905 participantes del "Estudio Poblacional de Salud Mental, Envigado 2017", recopilada a través de entrevistas realizadas con la World Health Organization Composite International Diagnostic Interview -CIDI-OMS. El procesamiento se realizó mediante el programa SPSS versión 23. Resultados: Cuatro factores explican la probabilidad de depresión mayor en el último año, en la población de estudio: el abuso de drogas diferentes al alcohol (ORaj=4,43 $[1,164-16,833])$, la muerte o enfermedad de un familiar cercano (ORaj=4,15 [1,583-10,880]), presentar altos niveles de resiliencia (ORaj=0,22 [0,112-0,425]) y una percepción excelente o buena de su salud mental (ORaj=0,19 [0,098-0,378]). Conclusiones: Los factores que aumentan la probabilidad de depresión mayor en la población de estudio son: abuso de drogas diferentes al alcohol y la muerte o enfermedad de un familiar de primer grado de 
consanguinidad; y los factores protectores son altos niveles de resiliencia y una percepción excelente o buena de la propia salud mental. Esta evidencia refuerza la necesidad de implementar programas de promoción centrados en el mejoramiento de habilidades para la vida, que propicien estrategias adecuadas frente a los desafíos.

Palabras clave: salud mental, trastornos mentales, depresión, resiliencia, drogas, epidemiología, Envigado.

\section{Abstract}

Introduction: Depression affects more than $\mathbf{3 0 0}$ million people in the world, it is the leading cause of disability and contributes significantly to the global burden of disease. Objective: To determine the prevalence and some social, personal, and family factors associated with major depressive disorder in a population aged from 15 to 65 in Envigado (Colombia) in 2017. Methods: A cross-sectional study with analytical intent was conducted, based on the information from 905 participants for the "Population study of mental health 2017", collected through interviews administered by means of the World Health Organization Composite International Diagnostic Interview -CIDI-OMS. The processing was performed using the SPSS program version 23. (CES University License). Results: Four factors correlate with major depression in the last year in the study population: drug abuse other than alcohol (ORaj $=4,43$ $[1,164-16,833])$, a close relative's death or illness (ORaj $=4,15[1,583-10,880]$ ), high resilience (ORaj $=0.22[0,112-0,425]$ ) and an excellent or good mental health perception (ORaj $=0.19$ [0.098-0.378]). Conclusions: the factors that increase the probability of major depression in the study population are drug abuse other than alcohol and the death or illness of a first-degree relative; and the protective factors are high levels of resilience and an excellent or good perception of one's own mental health. This evidence reinforces the need to implement promotion programs focused on improvement of life skills, which provide adequate strategies to face the challenges.

Keywords: mental health, mental disorders, depression, resilience, drugs, epidemiology, Envigado.

\section{Introducción}

La depresión es una enfermedad frecuente y se calcula que afecta a más de $\mathbf{3 0 0}$ millones de personas en el mundo. Es la principal causa de discapacidad y contribuye de forma muy importante a la carga mundial general de morbilidad (Organización Mundial de la Salud -OMS- 
2020). Según la OMS, en el año 2015 , el $4,4 \%$ de la población mundial sufría de un trastorno depresivo, mientras que en Colombia era de 4,7\% (World Health Organization -WHO-, 2017).

Los trastornos depresivos se caracterizan por una tristeza de una intensidad o una duración suficiente como para interferir en la funcionalidad de quien la padece y, en ocasiones, por una disminución del interés o del placer despertado por las actividades. Se desconoce la causa exacta, no obstante, diversos estudios señalan como posibles actores cambios en las concentraciones de neurotransmisores, una alteración en la función neuroendocrina y factores psicosociales (Coryell, 2020).

Según la cuarta edición del Manual Diagnóstico y Estadístico de las Enfermedades Mentales (DSM-4) ${ }^{1}$, la depresión mayor hace parte de los "trastornos afectivos" y se ubica dentro del subgrupo de trastornos depresivos (American Psychiatric Association, 1994). Se define como un trastorno del estado del ánimo en el cual predominan síntomas afectivos tales como tristeza profunda, apatía, anhedonia, desesperanza e irritabilidad, y puede acompañarse de síntomas cognitivos (Ministerio de Salud y Protección Social -Colombia-, 2017).

Se ha encontrado que los trastornos depresivos tienen mayor prevalencia e incidencia en mujeres que en hombres, ya que las primeras están más expuestas a factores estresantes (Hegeman et al., 2012). Así mismo, se ha observado que los pacientes con trastornos de ansiedad tienen un riesgo mayor de desarrollar un primer episodio de depresión mayor y aproximadamente $80 \%$ de las personas con depresión han experimentado un evento estresor importante en su vida (muerte de un ser querido, crisis financiera, infidelidad de la pareja, discusiones, rupturas, entre otros) (Hammen, 2018).

Se ha observado, además, que la depresión puede relacionarse con abuso de alcohol, enfermedades sistémicas, trastornos alimentarios y de la personalidad, y en mayor medida es uno de los factores asociados con conductas suicidas, siendo el riesgo cuatro veces mayor en las personas con depresión que en la población en general (Ministerio de Sanidad, Servicios Sociales e Igualdad -España-, 2014).

Además de los factores de riesgo, existen diversos factores protectores de la depresión, tales como la actividad física, las buenas relaciones con los demás y la resiliencia, entendida esta última como una respuesta positiva que se activa en un estado de estrés, con el fin de prevenir el desarrollo de desenlaces negativos en la salud mental (Anyan \& Hjemdal, 2016). En el

\footnotetext{
${ }^{1}$ Para el presente estudio se tomó como base la definición de depresión mayor de la clasificación DSM-4, dado que el instrumento de recolección de información fue la encuesta CIDI-CAPI, la cual incluye dicha clasificación en los algoritmos que determinan los diagnósticos.
} 
presente estudio se hizo énfasis en este último factor, debido a la relevancia que ha venido cobrando en los estudios científicos (Poole, Dobson, \& Pusch, 2017).

Dada la importancia y la necesidad de identificar los factores de riesgo y protección asociados a los trastornos depresivos para la implementación de programas de promoción de la salud mental (Fundación Victoria para la Promoción de Salud et al., 2004; Jané-Llopis, 2004; OMS, 2005), el objetivo de esta investigación fue determinar la prevalencia de la depresión mayor y su relación con algunos factores personales, sociales y familiares, en la población de 15 a 65 años del municipio de Envigado (Colombia).

\section{Método}

Se realizó un estudio analítico cross-sectional representativo de la población de 15 a 65 años, residente en el municipio de Envigado (Colombia). Para el análisis se tomó la información del "Estudio Poblacional de Salud Mental, Envigado 2017" realizado por el Centro de Excelencia de Investigación en Salud Mental (CESISM) de la Universidad CES y la Secretaría de Salud de la Alcaldía de Envigado (2018). La información del estudio primario fue recopilada a través de la aplicación de entrevistas estructuradas en el hogar, mediante la World Health Organization Composite International Diagnostic Interview (versión CIDI-CAPI 3.0), instrumento de la OMS, que fue adaptado culturalmente en la ciudad de Medellín y que permite la generación de diagnósticos de trastornos mentales, entre ellos, el trastorno de depresión mayor (Haro et al., 2006), a partir de la clasificación de enfermedades psiquiátrica del DSM-IV². Además, el CIDI indaga información respecto a datos demográficos, condiciones crónicas de salud, eventos vitales, red de apoyo, consumo, abuso y dependencia del alcohol y de otras sustancias psicoactivas.

La valoración de la resiliencia a través de la CIDI corresponde a la escala construida por Wagnild y Young (1993), compuesta de 25 ítems que puntúan en una escala tipo Likert de 7 puntos, en la que 1 es en desacuerdo y 7 es un máximo de acuerdo. Cubren cinco áreas o perspectivas de respuesta: satisfacción personal, ecuanimidad, sentirse bien solo, confianza en sí mismo, perseverancia y satisfacción personal, y un puntaje entre 25 y 175 será indicador de mayor resiliencia. El alfa de Cronbach de la escala fue de 0.89 . El puntaje total es codificado como alto, moderado o bajo, siendo los puntajes altos indicadores de mayor resiliencia.

\footnotetext{
${ }^{2}$ Aún no se cuenta con la versión CIDI basada en el DSM-5 (Navarro-Mateu et al., 2013).
} 
Por otro lado, la información respecto a la red de apoyo social que recoge la CIDI contempla 10 ítems que hacen referencia a la relación con la familia y los amigos.

La CIDI fue aplicada por trabajadores sociales y personal con experiencia en aplicación de entrevistas. El entrenamiento sobre el manejo de la CIDI, recomendaciones para abordar la entrevista, formular las preguntas, identificar respuestas y situaciones problemáticas, estuvo a cargo del Grupo de Investigación en Salud Mental de la Universidad CES.

\section{Población y muestra}

La población de estudio estuvo constituida por población civil no institucionalizada, e incluyó adolescentes (15 a 18 años) y adultos (19 a 65 años); el tamaño de la muestra del estudio primario fue calculado con base en la fórmula de estimación de una proporción poblacional, para lo cual se utilizó un nivel de confianza del 95\%, una precisión del 5\% y una prevalencia estimada del 9,95\% (prevalencia de vida esperada para el diagnóstico de depresión mayor según resultados del Primer Estudio de Salud Mental - Medellín 2012; The WHO World Mental Health Survey Consortium et al., 2012). El proceso de selección de la muestra fue probabilístico, multietápico, a partir del marco muestral que comprendía la totalidad de las viviendas de la ciudad de Medellín ubicadas tanto en la zona urbana como rural. El tamaño de la muestra a la que finalmente se aplicó la entrevista en el estudio primario fue de 2.072 personas, quienes previamente confirmaron su participación por medio de la firma de los respectivos asentimientos (menores de edad) y consentimientos informados.

Para el desarrollo del presente análisis, se incluyó la información de todos los participantes de la base de datos del estudio primario antes mencionado, correspondientes a la población que había dado positivo para algún trastorno mental (905 personas); es de resaltar que se tomaron medidas de control de calidad de la información, correspondientes a la selección y depuración de las variables requeridas para el análisis.

\section{Análisis estadístico}

Con el fin de describir los factores perso-nales, sociales y familiares de quienes conformaron la muestra, se obtuvieron frecuencias absolutas y relativas para las variables cualitativas.

Posteriormente, se calcularon las prevalencias de vida y año del trastorno de de-presión mayor con sus respectivos intervalos de confianza según las variables edad, sexo y zona de residencia como ejes principales de análisis. Se generaron tablas de contingencia tomando el trastorno depresivo mayor en el último año como variable dependiente y los facto־res personales, 
sociales y familiares como variables independientes. En el análisis se llevaron a cabo las pruebas estadísticas Chi-Cuadrado de Independencia y el Test Exacto de Fisher; además, se realizó el cálculo de las medidas de fuerza de asociación OR (Odds Ratio) crudas con intervalos de confianza del 95\%. Final-mente, se realizó un modelo multivariado de regresión logística binaria con el propósito de ajustar la probabilidad de trastorno de depresión mayor de la población con potenciales variables de confusión que en el análisis bivariado se encontraron relacionadas y que fueron significativas a un nivel de significación es-tadística de 0,25 según el criterio de Hosmer-Lemeshow; estas variables fueron: la percepción de salud mental, la muerte o enfermedad de un familiar de primer grado, los problemas graves con un amigo o vecino, una crisis financiera reciente, la pérdida o robo de algo importante, la presencia de resiliencia, el abuso de alcohol, el abuso de drogas y la dependencia de drogas. El procesamiento de información se realizó en el programa SPSS ${ }^{\circledR}$ versión 23 (licencia Universidad CES).

\section{Consideraciones éticas}

El estudio fue avalado por el comité de ética de la Universidad CES y de acuerdo con sus características se clasificó como una investigación sin riesgo, con base en el artículo 11, numeral a), de la Resolución 8430 de 1993, de la Dirección de desarrollo científico y tecnológico del entonces Ministerio de Salud de Colombia. Con base en los principios éticos de la investigación (respeto por las personas, bene-ficencia y justicia), los resultados obtenidos no fueron utilizados para fines distintos a los de determinar la prevalencia de la depresión mayor en la población de Envigado y los factores personales, familiares y sociales asociados.

\section{Resultados}

Se analizó la población general del municipio de Envigado (Colombia) a partir de una muestra de 905 encuestados con edades entre 15 y 65 años. El 58,2\% correspondía a mujeres y 22,2\% eran adolescentes. En correspondencia con la distribución de la población del municipio de Envigado, $82,1 \%$ de las encuestas se aplicaron en zona urbana y el porcentaje restante en zona rural. En cuanto a la prevalencia de depresión mayor, 13,5\% de los participantes reportaron que habían presentado este diagnóstico alguna vez en su vida y 5,4\% durante los 12 meses anteriores al estudio.

\section{Factores personales y consumo de drogas}

El diagnóstico de depresión mayor se presentó en mayor proporción en mujeres que en hombres $(6,1 \%$ vs $4,5 \%)$ y en adolescentes respecto al grupo de adultos $(6,5 \%$ vs $5,1 \%)$. Al analizar la relación entre depresión mayor y algunos factores personales como sexo, edad, área de residencia y percepción de salud física y mental, se encontró asociación estadísticamente 
significativa con el último factor ( $O R=0,15 ; \mathrm{IC}: 0,077-0,279)$, de modo que las personas que tenían buena o excelente percepción de su salud mental presentaron aproximadamente $85 \%$ menos probabilidad de tener depresión, respecto a las personas que percibían su salud mental como regular o mala (Ver Tabla 1).

Respecto al consumo de alcohol, la prevalencia de depresión mayor fue 5 veces mayor en quienes lo habían consumido en el último año, 2 veces mayor en quienes habían abusado de esta sustancia y casi 3 veces en quienes presentaban dependencia. En cuanto al consumo de otras sustancias psicoactivas (marihuana, cocaína, heroína, entre otros), la prevalencia de depresión mayor fue mayor en quienes abusaban de estas sustancias respecto a los que no $(13,0 \%$ vs 5,0\%, respectivamente). Se encontró asociación estadísticamente significativa entre el diagnóstico de depresión mayor y el abuso de alcohol (valor $p=0,01)$ y el abuso de otras sustancias psicoactivas diferentes al alcohol (valor $p=0,01$ ), de modo que las personas que abusaban de estas otras sustancias tenían aproximadamente 3 veces más la probabilidad de presentar depresión respecto a las que no $(\mathrm{OR}=2,85 ;$ IC: 1,144 - 7,080) (ver Tabla 2). 
Tabla 1. Factores personales y eventos estresantes asociados al trastorno de depresión mayor en la población de estudio.

\begin{tabular}{|c|c|c|c|c|c|c|}
\hline \multirow{3}{*}{$\begin{array}{l}\text { Factores personales y eventos } \\
\text { vitales estresantes }\end{array}$} & \multicolumn{4}{|c|}{ Depresión mayor } & \multirow{3}{*}{ Valor $p$} & \multirow{3}{*}{ OR cruda- IC 95\% } \\
\hline & \multicolumn{2}{|c|}{ Si } & \multicolumn{2}{|c|}{ No } & & \\
\hline & $N$ & $\%$ & No & $\%$ & & \\
\hline Sexo & & & & & 0,301 & \\
\hline Hombre & 17 & $4,50 \%$ & 361 & $95,50 \%$ & & 1 \\
\hline Mujer & 32 & $6,10 \%$ & 495 & $93,90 \%$ & & $0,728(0,399-1,332)$ \\
\hline Grupo de edad & & & & & 0,454 & \\
\hline Adolescentes & 13 & $6,50 \%$ & 188 & $93,50 \%$ & & $1,283(0,667-2,469)$ \\
\hline Adultos & 36 & $5,10 \%$ & 668 & $94,90 \%$ & & 1 \\
\hline Percepción de salud mental & & & & & 0.000 & \\
\hline Buena-Excelente & 32 & $3,90 \%$ & 794 & $96,10 \%$ & & 1 \\
\hline Regular-Mala & 17 & $21,50 \%$ & 62 & $78,50 \%$ & & $0,146(0,077-0,279)$ \\
\hline Percepción de salud física & & & & & 0,058 & \\
\hline Buena-Excelente & 38 & $4,90 \%$ & 745 & $95,10 \%$ & & 1 \\
\hline Regular-Mala & 11 & $9 \%$ & 111 & $91 \%$ & & $0,514(0,256-1,037)$ \\
\hline Área de residencia & & & & & 0,148 & \\
\hline Urbana & 44 & $5,90 \%$ & 699 & $94,10 \%$ & & $1,97(0,771-5,065)$ \\
\hline Rural & 5 & $3,10 \%$ & 157 & $96,90 \%$ & & 1 \\
\hline Muerte de familiar de primer $g$ & & & & & 0,044 & \\
\hline $\mathrm{Si}$ & 6 & $11,50 \%$ & 46 & $88,50 \%$ & & $2,457(0,995-6,070)$ \\
\hline No & 43 & $5,00 \%$ & 810 & $95,00 \%$ & & 1 \\
\hline $\begin{array}{l}\text { Problemas serios con un amigo } \\
\text { cercano, vecino o familiar }\end{array}$ & & & & & 0,001 & \\
\hline $\mathrm{Si}$ & 7 & $16,30 \%$ & 36 & $83,70 \%$ & & $3,792(1,593-9,023)$ \\
\hline No & 42 & $4,90 \%$ & 819 & $95,10 \%$ & & 1 \\
\hline $\begin{array}{l}\text { Desempleo o en busca de trabc } \\
\text { por } 1 \text { mes sin éxito }\end{array}$ & & & & & 0,056 & \\
\hline $\mathrm{Si}$ & 10 & $9,30 \%$ & 97 & $90,70 \%$ & & $2,004(0,969-4,142)$ \\
\hline No & 39 & $4,90 \%$ & 758 & $95,10 \%$ & & 1 \\
\hline $\begin{array}{l}\text { Crisis financiera grave o proble } \\
\text { económicos importantes }\end{array}$ & & & & & 0,008 & \\
\hline $\mathrm{Si}$ & 13 & $10,40 \%$ & 112 & $89,60 \%$ & & $2,396(1,232-4,656)$ \\
\hline No & 36 & $4,60 \%$ & 743 & $95,40 \%$ & & 1 \\
\hline $\begin{array}{l}\text { Pérdida o robo de algo de muc } \\
\text { valor }\end{array}$ & & & & & 0,035 & \\
\hline $\mathrm{Si}$ & 4 & $14,30 \%$ & 24 & $85,70 \%$ & & $3,078(1,024-9,248)$ \\
\hline No & 45 & $5,10 \%$ & 831 & $94,90 \%$ & & 1 \\
\hline
\end{tabular}


Tabla 2. Consumo de drogas y trastorno de depresión mayor en la población de estudio.

\begin{tabular}{|c|c|c|c|c|c|c|}
\hline \multirow{3}{*}{ Factores personales } & \multicolumn{4}{|c|}{ Depresión mayor } & \multirow{3}{*}{ Valor $p$} & \multirow{3}{*}{ OR cruda- IC $95 \%$} \\
\hline & \multicolumn{2}{|r|}{$\mathrm{Si}$} & \multicolumn{2}{|c|}{ No } & & \\
\hline & $\mathbf{N}$ & $\%$ & No & $\%$ & & \\
\hline Consumo de alcohol & & & & & 0,052 & \\
\hline Si & 48 & $5,90 \%$ & 760 & $94,10 \%$ & & $6,063(0,872-44,427)$ \\
\hline No & 1 & $1,00 \%$ & 96 & $99,00 \%$ & & 1 \\
\hline Abuso de alcohol & & & & & 0,019 & \\
\hline $\mathrm{Si}$ & 10 & $10,50 \%$ & 85 & $89,50 \%$ & & $2,326(1,121-4,826)$ \\
\hline No & 39 & $4,80 \%$ & 771 & $95,20 \%$ & & 1 \\
\hline Dependencia de alcohol & & & & & 0,081 & \\
\hline $\mathrm{Si}$ & 4 & $12,90 \%$ & 27 & $87,10 \%$ & & $2,729(0,916-8,134)$ \\
\hline No & 45 & $5,10 \%$ & 829 & $94,90 \%$ & & 1 \\
\hline Abuso de drogas & & & & & 0,018 & \\
\hline Si & 6 & $13,00 \%$ & 40 & $87,00 \%$ & & $2,847(1,144-7,080)$ \\
\hline No & 43 & $5 \%$ & 816 & $95 \%$ & & 1 \\
\hline Dependencia de drogas & & & & & 0,05 & \\
\hline Si & 3 & $18,80 \%$ & 13 & $81,30 \%$ & & $4,229(1,164-15,363)$ \\
\hline No & 46 & $5,20 \%$ & 843 & $94,80 \%$ & & 1 \\
\hline
\end{tabular}

\section{Eventos vitales estresantes}

La presencia de diagnóstico de depresión mayor presentó asociación estadísticamente significativa con las variables de muerte o enfermedad de un familiar de primer grado de consanguinidad (valor $p=0,044$ ), crisis financiera reciente (valor $p=0,008$ ), pérdida o robo de algo de mucho valor (valor $\mathrm{p}=0,035$ ), y presencia de problemas serios con un amigo cercano, vecino o familiar (valor $p=0,001$ ), de modo que la probabilidad de depresión mayor en quienes tenían estos problemas contenidos en la última variable, era 4 veces mayor respecto a la probabilidad de quienes no los tenían (OR=3,79; IC: 1,593-9,023) (ver Tabla 1).

\section{Depresión mayor y resiliencia}

Se encontró asociación estadísticamente significativa entre los niveles de resiliencia y el diagnóstico de depresión mayor (valor $p=0,000$ ), de modo que la prevalencia anual del diagnóstico en quienes presentaron resiliencia baja o moderada fue casi 5 veces mayor respecto a quienes presentaron niveles altos de resiliencia ( $11,1 \%$ vs $2,4 \%$ ).

Al ajustar la probabilidad de presentar trastorno de depresión mayor por los nueve factores potenciales de confusión identificados en el análisis bivariado, se identificaron cuatro factores que explican dicha probabilidad: presentar abuso de drogas diferentes al alcohol (ORaj=4,43 $[1,164-16,833])$, muerte o enfermedad de un familiar cercano (ORaj=4,15 $[1,583-10,880]$ ), 
presentar altos niveles de resiliencia (ORaj=0,22 [0,112-0,425]) y tener una percepción excelente o buena de la propia salud mental (ORaj=0,19 [0,098-0,378]); los dos primeros factores aumentan la probabilidad de depresión mayor y los dos últimos la disminuyen (ver Tabla 4).

Tabla 4. Factores personales, sociales y familiares asociados al trastorno de depresión mayor en la población de estudio. Comparativo OR crudas vs OR ajustadas.

\section{Factores}

Percepción de salud mental

Buena - excelente*

Regular -mala

Muerte o enfermedad de un familiar de primer

$\mathrm{Si}$

No*

Resiliencia

Alta*

Baja-moderada

Abuso de drogas

$\mathrm{Si}$

No*
Depresión mayor

OR cruda-IC 95\%

1

$0,146(0,077-0,279)$

2,457 (1,000-6,070)

1

1

$0,193(0,102-0,365)$

$2,847(1,144-7,080)$

1
OR ajustada-IC 95\%

1

$0,192(0,098-0,378)$

$4,150(1,583-10,880)$

1

1

$0,218(0,112-0,425)$

$4,427(1,164-16,833)$

1

\section{Discusión}

Según la OMS, la depresión es un trastorno mental frecuente que se caracteriza por la presencia de tristeza, pérdida de interés o placer, falta de autoestima, culpa, alteraciones del sueño o del apetito, sensación de cansancio y falta de concentración (s. f.). Actualmente, cerca de 322 millones de personas a nivel mundial viven con depresión y se estima que para el 2020 será la segunda causa de discapacidad a nivel mundial, y la primera causa en países en desarrollo (Navarro-Loli et al., 2017). En Colombia, según el Ministerio de Salud y Protección Social, los casos de depresión han venido en aumento desde el año 2009, por lo que se considera un problema de salud pública (2017).

Uno de los principales hallazgos del "Estudio Poblacional de Salud Mental, Envigado 2017" fue la alta prevalencia anual de depresión mayor (5.4\%) (Centro de Excelencia en Investigación en Salud Mental de la Universidad CES \& Secretaria de Salud de la Alcaldía de Envigado, 2018), comparada con las prevalencias de este mismo diagnóstico reportadas en otros estudios como el de la OMS, que informa una prevalencia a nivel mundial de 4.4\% (WHO, 2017). En el ámbito local, el estudio realizado en el municipio de Itagüí (aledaño al de Envigado) reportó una prevalencia de $2.7 \%$ en el año 2011 (Castaño, Pérez, \& Sierra Hincapié, 2016) y en la ciudad de 
Medellín (aledaña al municipio de Envigado) para el periodo 2011-2012 de 4.10\% (The WHO World Mental Health Survey Consortium et al., 2012). La prevalencia encontrada en la población de Envigado es igual a la prevalencia a nivel nacional (Colombia) que fue de $5.4 \%$ (Ministerio de Salud y Protección Social -Colombia-, 2015) y similar a la reportada en las Américas correspondiente a 5\%, para el 2015 (Organización Panamericana de la Salud \& Organización Mundial de la Salud, 2017).

La prevalencia del diagnóstico de depresión mayor en el municipio de Envigado (Colombia) según el sexo fue mayor en mujeres (6.10\%) que en hombres (4,50\%); pese a que dicha diferencia no fue significativa, coincide con la tendencia general respecto a que la depresión es un trastorno que afecta en mayor medida a las mujeres desde la adolescencia hasta la edad adulta (Hegeman et al., 2012). Según la OMS, la prevalencia de depresión en mujeres es de 5,1\% y en hombres $3,6 \%$ y la tasa de prevalencia según la edad alcanza su punto máximo en personas mayores (superior a 7,5\% entre las mujeres de 55-74 años, y más de 5.5\% entre los hombres) (WHO, 2017). En el estudio de Salud Mental Medellín 2011-2012 la prevalencia fue de 1.62 en hombres y 5.56 en mujeres, que equivale a una proporción de 3,4 mujeres por cada hombre.

En el presente estudio se encontró que los factores que aumentan la probabilidad de presentar depresión mayor en la población del municipio de Envigado (Colombia) son el abuso de drogas diferentes al alcohol y la muerte o enfermedad de un familiar de primer grado de consanguinidad. En cuanto a los factores protectores, se encontró que altos niveles de resiliencia y tener una percepción excelente o buena de la salud mental, disminuyen dicha probabilidad.

La depresión es una de las principales causas del consumo de sustancias psicoactivas, especialmente en los adolescentes, sin embargo, también puede ser consecuencia del consumo; está doble condición genera una situación muy compleja por la cual este comportamiento se constituye en uno los mayores problemas de salud pública en las sociedades actuales (Butler et al., 2006; Gómez Rodríguez et al., 2013; Masten, 2014; Ministerio de Sanidad, Servicios Sociales e Igualdad -España-, 2014). En el presente estudio se encontró una asociación significativa entre depresión y abuso de alcohol y sustancias psicoactivas; de manera que quienes abusan del alcohol y de sustancias psicoactivas presentan hasta tres veces más de probabilidad de padecer este trastorno. En esta línea, un estudio realizado en la ciudad de Bogotá reportó que las mujeres con síntomas depresivos eran las que más consumían cigarrillo, y tanto los hombres como las mujeres que consumían alcohol padecían más frecuentemente de depresión (Gómez Rodríguez et al., 2013).

Adicionalmente, la comorbilidad entre los trastornos mentales y los relacionados con el consumo de sustancias, aumenta el riesgo de presentar conductas violentas, lo que implica más riesgo de intentos suicidas y heteroagresión (Claro et al., 2015). 
Respecto a la muerte o enfermedad de un familiar cercano como factor de riesgo de la depresión mayor, San Molina (2010) encontró que la depresión es más frecuente en los seis meses posteriores a un suceso estresante como la muerte de un ser querido, entre otros.

La resiliencia, definida como la capacidad de adaptación para hacer frente a la adversidad o al trauma (Bonanno, 2004; Rutter, 1985) y facilitada por un buen apoyo social (Earvolino-Ramirez, 2007), según Poole, Dobson y Pusch (2017), es un factor protector frente a la posibilidad de tener un trastorno depresivo y, además, ofrece al individuo la capacidad de resolver y afrontar problemas. Se ha evidenciado que los pacientes con mayor resiliencia presentan menores síntomas depresivos, mientras que un grado medio de resiliencia favorece la aparición de un trastorno depresivo mayor (Agudelo, Antes, \& Torres, 2017). Lo anterior coincide con los hallazgos del presente estudio según los cuales las personas con depresión mayor presentaron baja o moderada resiliencia.

Así mismo, en el presente estudio las personas que tenían menor probabilidad de tener depresión tenían una buena o excelente percepción de su salud mental comparadas con aquellas que percibían regular o mala su salud mental. Con respecto a la percepción de salud mental, está demostrado que ésta afecta la salud física (Ministerio de Salud y Protección Social -Colombia-, 2015); lo que probablemente incide en la percepción de un mayor bienestar general e interés por realizar diversas actividades, actuando como factor protector frente a la depresión.

Finalmente, en cuanto al comportamiento de eventos vitales como las enfermedades (Serrano, 2002), heridas o agresiones graves, divorcio (Cedeño Pinzon, 2015) o separación y desempleo (Leonardi, 2019), en el presente estudio no presentaron asociación con la depresión mayor; es decir, estos factores no presentan importantes diferencias entre las personas diagnosticadas con depresión y las que no, en la población de 15 a 65 años del municipio de Envigado. No obstante, en otros estudios se encontró que el maltrato se asocia a una mayor frecuencia de episodios depresivos previos, mayor intensidad de la sintomatología depresiva, tendencia a la autodevaluación, conducta suicida a lo largo de la vida, rasgos disfuncionales de la personalidad, funcionamiento académico/laboral más afectado, disfunciones cognitivas sutiles, paranoidismo y dificultades en la interacción social (Martínez et al., 2018; Mall et al., 2018).

Aunque en el presente estudio la variable desempleo no presentó asociación estadísticamente significativa con la depresión mayor, es importante resaltar que $9 \%$ de las personas que se encontraban sin empleo presentaron trastorno depresivo mayor; situación explicable dado que el desempleo afecta considerablemente el bienestar de las personas, genera sentimientos de apatía, resignación, dudas sobre sí mismo, baja autoestima y pensamientos negativos que finalmente pueden derivar en depresión (Fuhrimann, 2017). Según Leonardi, el desempleo se encuentra asociado a mayor cantidad de síntomas depresivos, afectando en gran proporción a 
los colombianos debido a las condiciones del mercado laboral del país (2019). Si se compara la razón de prevalencia de tensión psicológica no específica entre desempleados y empleados se encuentra una relación estrecha con el desempleo y un mayor riesgo de padecerla (Caicedo \& van Gameren, 2016).

El presente estudio permite comparar los hallazgos obtenidos en la población del municipio de Envigado con los de otras ciudades de Colombia y otros países del mundo; y a partir del análisis de estas comparaciones generar reflexiones, planes y acciones en salud mental.

\section{Limitaciones}

Debido a que el estudio fue de tipo transversal, no se puede afirmar causalidad entre los factores analizados y el diagnóstico de depresión mayor, y dado que se utilizó una fuente secundaria de información, existen variables que posiblemente explican la prevalencia del diagnóstico, pero no fueron analizadas porque no se incluyeron en el estudio primario.

Se recomienda realizar estudios de fuente primaria, en los cuales se profundicen aspectos relacionados con la dinámica familiar, aspectos económicos en adultos y relacionados con la relación con pares en jóvenes y adolescentes.

\section{Conclusiones}

Los factores que aumentan la probabilidad de presentar depresión mayor en la población del municipio de Envigado (Colombia) son: abuso de drogas diferentes al alcohol y la muerte o enfermedad de un familiar de primer grado de consanguinidad. En cuanto a los factores protectores, altos niveles de resiliencia y tener una percepción excelente o buena de la salud mental, disminuyen dicha probabilidad. Esta evidencia refuerza la necesidad de implementar programas de prevención centrados en el mejoramiento de habilidades para la vida.

\section{Financiación}

El estudio primario fue financiado por medio del Convenio de Asociación número 13-50-0931-025-17 suscrito entre la Secretaría de Salud de Envigado y la Universidad CES.

\section{Conflicto de intereses}

Los autores manifiestan que no existe conflicto de intereses.

\section{Agradecimientos}

A la doctora Gloria Sierra Hincapié, docente investigadora, quien en nombre del Centro de Excelencia en Investigación en Salud Mental (Universidad CES) apoyó el procesamiento de la base de datos. 


\section{Referencias}

Agudelo Martínez, M. A., Ante Chaves, C., \& Torres de Galvis, Y. (2016). Factores personales y sociales asociados al trastorno de depresión mayor, Medellín (Colombia), 2012. CES Psicología, 10(1), 21-34. https://doi.org/10.21615/cesp.10.1.2

American Psychiatric Association. (1994). Diagnostic and Statistical Manual of Mental Disorders Fourth Edition. https://justines2010blog.files.wordpress.com/2011/03/dsm-iv.pdf

Anyan, F., \& Hjemdal, O. (2016). Adolescent stress and symptoms of anxiety and depression: Resilience explains and differentiates the relationships. Journal of Affective Disorders, 203, 213-220. https://doi.org/10.1016/i.jad.2016.05.031

Bonanno, G. A. (2004). Loss, Trauma, and Human Resilience: Have We Underestimated the Human Capacity to Thrive After Extremely Aversive Events? American Psychologist, 59(1), 20-28. https://doi.org/10.1037/0003-066X.59.1.20

Butler, R., Carney, S., Cipriani, A., Geddes, J., Hatcher, S., Price, J., \& Von Korff, M. (2006). Depressive disorders. American Family Physician, 73(11), 1999-2004.

Caicedo, M., \& van Gameren, E. (2016). Desempleo y salud mental en la población de origen hispano en Estados Unidos: Un análisis epidemiológico. Ciência \& Saúde Coletiva, 21(3), 955-966. https://doi.org/10.1590/1413-81232015213.16592014

Castaño Pérez, G. A., \& Sierra Hincapié, G. M. (2016). Trastorno dual en población general de Itagüí, Colombia. Revista Colombiana de Psiquiatría, 45(2), 108-117. https://doi.org/10.1016/i.rcp.2015.08.005

Cedeño Pinzon, E.J. (2015). La depresión: los efectos psicológicos y problemas emocionales en el adulto, ante la etapa del divorcio. http://repositorio.utmachala.edu.ec/handle/48000/4163

Centro de Excelencia en Investigación en Salud Mental Universidad CES [CESCIM] \& Alcaldía de Envigado. (2018). Estudio Poblacional de Salud Mental Envigado 2017. http://hdl.handle.net/10946/3788

Claro, H. G., Oliveira, M. A. F. de, Titus, J. C., Fernandes, I. F. de A. L., Pinho, P. H., \& Tarifa, R. R. (2015). Drug use, mental health and problems related to crime and violence: Crosssectional study. Revista Latino-Americana de Enfermagem, 23(6), 1173-1180. https://doi.org/10.1590/0104-1169.0478.2663

Coryell, W. (2020). Trastornos depresivos-Trastornos psiquiátricos. Manual MSD versión para profesionales. $\quad$ https://www.msdmanuals.com/es-co/professional/trastornospsiqui\%C3\%A1tricos/trastornos-del-estado-de-\%C3\%A1nimo/trastornos-depresivos

Earvolino-Ramirez, M. (2007). Resilience: A Concept Analysis. Nursing Forum, 42(2), 73-82. https://doi.org/10.1111/i.1744-6198.2007.00070.x

Fuhrimann, C. M. (2017). La depresión: Un reto para toda la sociedad del que debemos hablar. Revista Cubana de Salud Pública, 43(2), 137-138. http://www.revsaludpublica.sld.cu/index.php/spu/article/view/999/875 
Fundación Victoria para la Promoción de Salud, Universidad de Melbourne, \& Organización Mundial de la Salud. (2004). Promoción de la Salud Mental: Conceptos, evidencia emergente, práctica, informe compendiado. Organización Mundial de la Salud.

Gómez Rodríguez, D. T., Gutiérrez Herrera, M., \& Londoño, S. P. (2013). Depresión y consumo de sustancias psicoactivas en adolescentes en la ciudad de Bogotá. Psychologia, 7(1), 45. https://doi.org/10.21500/19002386.1193

Hammen, C. (2018). Risk Factors for Depression: An Autobiographical Review. Annual Review of Clinical Psychology, 14(1), 1-28. https://doi.org/10.1146/annurev-clinpsy-050817$\underline{084811}$

Haro, J. M., Arbabzadeh-Bouchez, S., Brugha, T. S., De Girolamo, G., Guyer, M. E., Jin, R., Lepine, J. P., ... Kessler, R. C. (2006). Concordance of the Composite International Diagnostic Interview Version 3.0 (CIDI 3.0) with standardized clinical assessments in the WHO World Mental Health Surveys. International Journal of Methods in Psychiatric Research, 15(4), 167-180. https://doi.org/10.1002/mpr.196

Hegeman, J. M., Kok, R. M., van der Mast, R. C., \& Giltay, E. J. (2012). Phenomenology of depression in older compared with younger adults: Meta-analysis. British Journal of Psychiatry, 200(04), 275-281. https://doi.org/10.1192/bjp.bp.111.095950

Jané-Llopis, E. (2004). La eficacia de la promoción de la salud mental y la prevención de los trastornos mentales. Revista de la Asociación Española de Neuropsiquiatría, 89, 67-77.

Leonardi, S. (2019). Asociación entre depresión y desempleo: Un estudio transversal en Colombia.

https://repository.javeriana.edu.co/bitstream/handle/10554/42735/Proyecto\%20de\% 20grado\%20Stefano\%20Leonardi\%20.pdf?sequence $=1 \&$ isAllowed $=y$

Mall, S., Mortier, P., Taljaard, L., Roos, J., Stein, D. J., \& Lochner, C. (2018). The relationship between childhood adversity, recent stressors, and depression in college students attending a South African university. BMC Psychiatry, 18, 1-1. https://doi.org/10.1186/s12888-017-1583-9

Martínez, R. F., Kokoulina, E., Campos, X., Araújo, E. C., Fuertes, I. G., Pousada, A. R., \& Batán, P. V. (2018). Ecofenotipos en la depresión mayor: El papel del maltrato físico en la infancia. Rev. Asoc. Esp. Neuropsiq, 38(133), 75-98. file:///C:/Users/Usuario/Downloads/17028-17904-1-PB.pdf

Masten, A. S. (2014). Global Perspectives on Resilience in Children and Youth. Child Development, 85(1), 6-20. https://doi.org/10.1111/cdev.12205

Ministerio de Salud y Protección Social -Colombia-. (2015). Encuesta Nacional de Salud Mental 2015.

http://www.odc.gov.co/Portals/1/publicaciones/pdf/consumo/estudios/nacionales/CO 031102015-salud mental tomol.pdf

Ministerio de Salud y Protección Social -Colombia-. (2017). Boletín de salud mental Depresión. Subdirección de Enfermedades No Transmisibles. 
Ministerio de Sanidad, Servicios Sociales e Igualdad -España-. (2014). Guía de Práctica Clínica sobre el Manejo de la Depresión en el Adulto. http://www.guiasalud.es/GPC/GPC 534 Depresion Adulto Avaliat compl.pdf

Navarro-Loli, J. S., Moscoso, M., \& Calderon de la cruz, G. (2017). Research on depression in adolescents in Peru: A systematic review. Liberabit: Revista Peruana de Psicología, 23(1), 57-74. https://doi.org/10.24265/liberabit.2017.v23n1.04

Navarro-Mateu, F., Morán-Sánchez, I., Alonso, J., Tormo, M. J., Pujalte, M. L., Garriga, A., Aguilar-Gaxiola, S., \& Navarro, C. (2013). Cultural adaptation of the Latin American version of the World Health Organization Composite International Diagnostic Interview (WHO-CIDI) (v 3.0) for use in Spain. Gaceta Sanitaria, 27(4), 325-331. https://doi.org/10.1016/i.gaceta.2012.06.005

Organización Mundial de la Salud [OMS]. (2005). Legislación sobre salud mental y derechos humanos: Conjunto de guías sobre servicios y políticas de salud mental. Editores Médicos S.A., EDIMSA.

Organización Mundial de la Salud [OMS]. (2020). Depresión. https://www.who.int/es/newsroom/fact-sheets/detail/depression

Organización Mundial de la Salud. (s. f.). Depresión. WHO, World Health Organization. http://www.who.int/topics/depression/es/

Organización Panamericana de la Salud \& Organización Mundial de la Salud [OPS/OMS]. (2017, marzo 30). "Depresión: hablemos", dice la OMS, mientras la depresión encabeza la lista de causas de enfermedad https://www.paho.org/hq/index.php?option=com content\&view=article\&id=13102: de pression-lets-talk-says-who-as-depression-tops-list-of-causes-of-illhealth\&ltemid=1926\&lang=es

Poole, J. C., Dobson, K. S., \& Pusch, D. (2017). Childhood adversity and adult depression: The protective role of psychological resilience. Child Abuse \& Neglect, 64, 89-100. https://doi.org/10.1016/i.chiabu.2016.12.012

Rutter, M. (1985). Resilience in the Face of Adversity: Protective Factors and Resistance to Psychiatric Disorder. British Journal of Psychiatry, 147(6), 598-611. https://doi.org/10.1192/bjp.147.6.598

San Molina, L. (2010). Comprender la depresión. Editorial AMAT.

Serrano, F. (2002). Depresión en enfermedades médicas. https://recyt.fecyt.es/index.php/ASSN/article/download/5565/4578/0

The WHO World Mental Health Survey Consortium, Grupo de investigación en Salud Mental Universidad CES, The International Consortium Psychiatric Epidemiology Universidad de Harvard, \& Secretaría de Salud de Medellín. (2012). Primer estudio poblacional de salud mental, Medellín, 2011-2012. http://www.odc.gov.co/Portals/1/publicaciones/pdf/consumo/estudios/locales/CO031 052011-primer-estudio-poblacional-de-salud-mental-medellin,-2011---2012.pdf 
Wagnild, G. \& Young, H. (1993). Escala de resiliencia. https://es.slideshare.net/ipsiasinstitutopsiquiatrico/39013071escaladeresilienciadewagnildyyoung

World Health Organization [WHO]. (2017). Depression and Other Common Mental Disorders. http://apps.who.int/iris/bitstream/handle/10665/254610/WHO-MSD-MER-2017.2eng.pdf?sequence $=1$ 